\title{
Recombinant expression of different mutant K-ras gene in pancreatic cancer Bxpc-3 cells and its effects on chemotherapy sensitivity
}

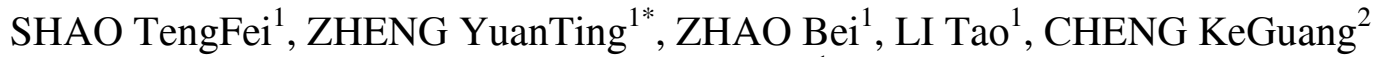 \\ \& CAI WeiMin ${ }^{1}$ \\ ${ }^{1}$ Department of Clinical Pharmacy, School of Pharmacy, Fudan University, Shanghai 201203, China; \\ ${ }^{2}$ Key Laboratory for the Chemistry and Molecular Engineering of Medicinal Resources, Ministry of Education of China, School of Chemistry \\ \& Chemical Engineering, Guangxi Normal University, Guilin 541004, China
}

Received June 28, 2014; accepted July 24, 2014; published online August 22, 2014

\begin{abstract}
$\mathrm{K}$-ras is a member of ras gene family which is involved in cell survival, proliferation and differentiation. When a mutation occurs in ras gene, the activation of Ras proteins may be prolonged to induce oncogenesis. However, the relationship between $\mathrm{K}$-ras mutation and clinical outcomes in pancreatic cancer patients treated with chemotherapy agents is still under debate. In this study, we constructed five pAcGFP1-C3 plasmids for different types of K-ras gene (WT, G12V, G12R, G12D, and G13D) and stably transfected human pancreatic cancer Bxpc-3 cells with these genes. The wild type and mutant clones showed a comparable growth and expression of K-Ras-GFP fusion protein. The expression of some K-ras mutations resulted in a reduced sensitivity to gefitinib, 5-FU, docetaxel and gemcitabine, while showed no effects on erlotinib or cisplatin. Moreover, compared with the wild type clone, K-Ras downstream signals (phospho-Akt and/or phospho-Erk) were increased in K-ras mutant clones. Interestingly, different types of K-ras mutation had non-identical K-Ras downstream signal activities and drug responses. Our results are the first to reveal the relationship between different K-ras mutation and drug sensitivities of these anti-cancer drugs in pancreatic cancer cells in vitro.
\end{abstract}

cancer chemotherapeutic, anti-cancer drug, K-ras, mutation, plasmids, pancreas

Citation: $\quad$ Shao TF, Zheng YT, Zhao B, Li T, Cheng KG, Cai WM. Recombinant expression of different mutant K-ras gene in pancreatic cancer Bxpc-3 cells and its effects on chemotherapy sensitivity. Sci China Life Sci, 2014, 57: 1011-1017, doi: 10.1007/s11427-014-4724-0

Pancreatic cancer is one of the most lethal human tumors [1], with a 5-year survival rate of less than $5 \%$. The lack of effective treatments is still one of the greatest challenges in clinical chemotherapy. A good prognostic marker for predicting the treatment response may improve the chemotherapeutic efficacy.

$\mathrm{K}$-ras mutation is found in $90 \%$ pancreatic adenocarcinomas [2,3], and the most common mutations are located at codon 12, 13, or 61 as shown in the Sanger Registry (http://www.sanger.ac.uk). Considering its important role in

*Corresponding author (email: zhengyuanting @ fudan.edu.cn) cell survival and proliferation, K-ras has been extensively studied in recent years. Previous studies revealed that K-ras gene mutations cause excessive activation of K-RAS/ RAF/MEK/ERK and PI3K/AKT/mTOR signaling pathways [4-9].

There are increasing evidences showing that K-ras mutational status can be regarded as a predictor of anti-epidermal growth factor (EGFR) monoclonal antibodies in colorectal cancer and non-small-cell lung cancer (NSCLC). Several recent phase 2 and 3 studies had demonstrated that mutant $\mathrm{K}$-ras could be regarded as a predictor of poor response to panitumumab and cetuximab in colorectal cancer [10]. 
However, the prognostic role of K-ras oncogenic mutation in patients who received cytotoxic chemotherapy is still under debate. Tsao et al. [11] reported that WT K-ras patients survived longer than K-ras mutant patients while treated with vinorelbine/cisplatin. Capelletti et al. [12] reported that patients with K-ras mutations had a worse prognosis and derived less benefit form adjuvant carboplatin/paclitaxel. However, Pickard et al. [13] and Rosty et al. [14] both proved that mutant K-ras did not correlate with disease progression while treated with 5-FU/folinic acid.

In the present study, we generated K-ras overexpressing clones with the four most common mutations (G12D, G12V, G12R and G13D) from human pancreatic cancer cell line Bxpc-3 with a wild-type (WT) egfr and K-ras. The chemotherapy sensitivity and its potential mechanism were also investigated in these clones to assess the predictive role of $\mathrm{K}$-ras status in cytotoxic chemotherapy in pancreatic cancer.

\section{Materials and methods}

\subsection{K-Ras-pAcGFP1-C3 plasmid construction}

The full sequence of K-ras gene carrying BamH I and Xho I sites, synthetized by Genewiz (Shanghai, China), was inserted into pAcGFP1-C3 vector (Clontech Laboratories, Inc, Mountain View, CA, USA) to construct K-Ras-pAcGFP1C3. K-ras gene and pAcGFP1-C3 vector were digested by BamH I and Xho I restriction enzyme, followed by isolation and purification, and then ligated with T4 DNA ligase. Ligation products were transformed to $E$. coli $\mathrm{DH} 5 \alpha$, and then spread on $2 \times$ YT medium culture plate with kanamycin, which were cultured at $37^{\circ} \mathrm{C}$ overnight. Positive clones were picked up and screened by PCR, identified via digesting by Bam $\mathrm{H}$ I and Xho I restriction enzyme and sequencing the inserted fragments.

\subsection{Transfection and screening Bxpc-3 cells with stable expression of K-ras}

Bxpc-3 cells (Chinese Academy of Sciences, Shanghai, China) were cultured in RPMI1640 (Gibco, Carlsbad, CA, USA) supplemented with $10 \%$ FBS (Gibco) at $37^{\circ} \mathrm{C}$ in a humidified atmosphere of a $5 \% \mathrm{CO}_{2}$. Bxpc-3 cells express wild-type (WT) egfr and K-ras, which can be obtained from the Wellcome Trust Sanger Institute Catalogue of Somatic Mutations in Cancer (COSMIC) website (http://www.sanger. ac.uk/cosmic/). $1 \times 10^{6}$ cells were seeded in a 12 -well plate, and $12 \mathrm{~h}$ later, plasmids were transfected into cells with lipofectamine 2000 (Invitrogen, Carlsbad, CA, USA) in RPMI1640 without FBS according to the manufacturer's instructions. Forty-eight hours after transfection, the cells were diluted and selected in medium containing G418 (200 $\mu \mathrm{g} \mathrm{mL}^{-1}$ ) for $14 \mathrm{~d}$ and then cloned using limiting dilution.
The selected clones were screened by Western blot and mixed clones from at least three independent clones were used in the following experiments.

\subsection{Growth curves analysis}

Cell growth curve was used to analyze the effect of overexpression of K-ras on the proliferation of Bxpc-3 cells. The cells were seeded in a 96-well plate with a destiny of 5000 cells each well. The cell number was detected every day by MTT assay as described before [15]. The absorbance was detected at $570 \mathrm{~nm}$ using a Multiskan GO microplate reader (Thermo Fisher Scientific Inc., Waltham, MA, USA).

\subsection{Drug sensitivity assay}

To compare the drug sensitivity in the established mutant clones, the following chemotherapy agents were used: erlotinib (100 mmol L ${ }^{-1}$ in DMSO), gefitinib $\left(20 \mathrm{mmol} \mathrm{L}^{-1}\right.$ in DMSO), fluorouracil (5-FU, $100 \mathrm{mmol} \mathrm{L}^{-1}$ in $\mathrm{H}_{2} \mathrm{O}$ ), docetaxel $\left(100 \mathrm{mmol} \mathrm{L}^{-1}\right.$ in alcohol), gemcitabine $\left(100 \mathrm{mmol} \mathrm{L}^{-1}\right.$ in water) and cisplatin (100 mmol $\mathrm{L}^{-1}$ in DMSO). Cells were seeded in 96-well plates as a density of 8000 cells per well and grew for $24 \mathrm{~h}$. They were then exposed to various concentrations of chemotherapy agents for $48 \mathrm{~h}$. The cytotoxicity effects of each drug on the growth of Bxpc-3 derived K-ras mutant clones were evaluated using MTT assay. Data are presented as mean $\pm \mathrm{SD}$ of three independent experiments.

\subsection{Apoptosis analysis}

Bxpc-3 cells were cultured in 6-well plates and treated with different chemotherapeutic agents for $48 \mathrm{~h}$. Apoptotic cells were then identified by dual staining with FITC-conjugated with Annexin $\mathrm{V}$ and propidium iodide (PI) following the manufacturer's protocol (BD biosciences, San Jose, CA, USA). Briefly, the collected cells were washed twice with PBS. Then, cells were incubated with Annexin V-FITC and then PI for $15 \mathrm{~min}$ in the dark at room temperature. Cells were then analyzed within $1 \mathrm{~h}$ using flow cytometry (FACS Calibur, Becton Dickinson, Franklin Lakes, NJ, USA) and a computer station running Cell-Quest software (Becton Dickinson, Franklin Lakes, NJ, USA). Meanwhile, stained cells were captured by a cooled CCD camera (Leica DMI4000D, Wetzlar, Germany) coupled to the microscope.

\subsection{Western blot analysis}

For Western blot analysis, cells were cultured with RPMI1640 with $0.1 \%$ FBS for $24 \mathrm{~h}$, followed by stimulated by $10 \%$ FBS for $30 \mathrm{~min}$. Protein was extracted using RIPA lysis buffer (Beyotime, Shanghai, China) with PMSF (final concentration $1 \mathrm{mg} \mathrm{mL}^{-1}$; Beyotime) and Protease Inhibitor Cocktail Set III (final concentration $5 \mu \mathrm{L} \mathrm{mL}{ }^{-1}$; Merck 
$\mathrm{KGaA}$, Darmstadt, Germany), followed by boiling at $95^{\circ} \mathrm{C}$ for 10 min with SDS-PAGE loading buffer (Beyotime). The proteins were separated in a gel containing $10 \%$ acrylamide. After electrophoresis, the separated proteins were transferred to PVDF membranes using $200 \mathrm{~mA}$ for $30 \mathrm{~min}$ (K-Ras and GFP) or 60 min ( $\beta$-Actin, Akt and Erk) according to the molecular weight of proteins. The membranes were blocked with 5\% BSA in Tris-buffered saline with $0.1 \%$ Tween 20 for $1 \mathrm{~h}$. After that, they were incubated with primary antibodies overnight at $4^{\circ} \mathrm{C}$. The following antibodies were used in our experiments: monoclonal mouse anti-phospho-Erk (Thr 202/Tyr 204), monoclonal rabbit anti-phospho-Akt (Ser473) antibodies (Cell Signaling Technology, Danvers, MA, USA); monoclonal mouse anti-GFP for both GFP (27 kD) and K-Ras-GFP fusion protein (48 kD), monoclonal rabbit anti-Akt, monoclonal rabbit anti-Erk antibodies (Beyotime); monoclonal mouse anti-K-Ras (Santa Cruz Biotechnology, Santa Cruz, CA, USA). After washing in TBST for four times, the membranes were incubated with secondary antibodies for $1 \mathrm{~h}$. After that, the membranes were washed again in TBST for three times, followed by detecting using ECL plus (Beyotime). Images were captured with the Alpha Innotech imaging system (CA, USA).

\subsection{Statistical analysis}

Data are expressed as the mean \pm SD with three replicates at least. One-way ANOVA followed by LSD multiple comparison was used to compare cell response to drugs between different groups, and $P<0.05$ was considered to be significant.

\section{Results}

\subsection{Stable expression of different types of mutant $\mathrm{K}-$ ras in Bxpc-3 cells}

The different types of K-ras cDNA were successfully inserted into pAcGFP1-C3 plasmid $(5.2 \mathrm{~kb})$ identified by BamH I and Xho I digestion and electrophoresis. The amplification product of full-length K-ras gene (576 bp) from $\mathrm{K}-\mathrm{Ras}-\mathrm{pAcGFP} 1-\mathrm{C} 3$ plasmid was also verified by agarose gel.

Plasmids with WT and four mutant K-ras genes were successfully transfected into human pancreatic cancer cell line Bxpc-3. Western blot analysis indicated that the K-RaspAcGFP1-C3 transfected cells expressed a $48 \mathrm{kD}$ K-RasGFP fusion protein, while the vector transfected cells expressed solely GFP protein $(27 \mathrm{kD})$ (Figure $1 \mathrm{~A})$. In addition, Bxpc-3 also showed endogenous WT K-ras expression $(21 \mathrm{kD})$, and overexpressed K-Ras-GFP fusion protein did not seem to change the expression of endogenous K-Ras.
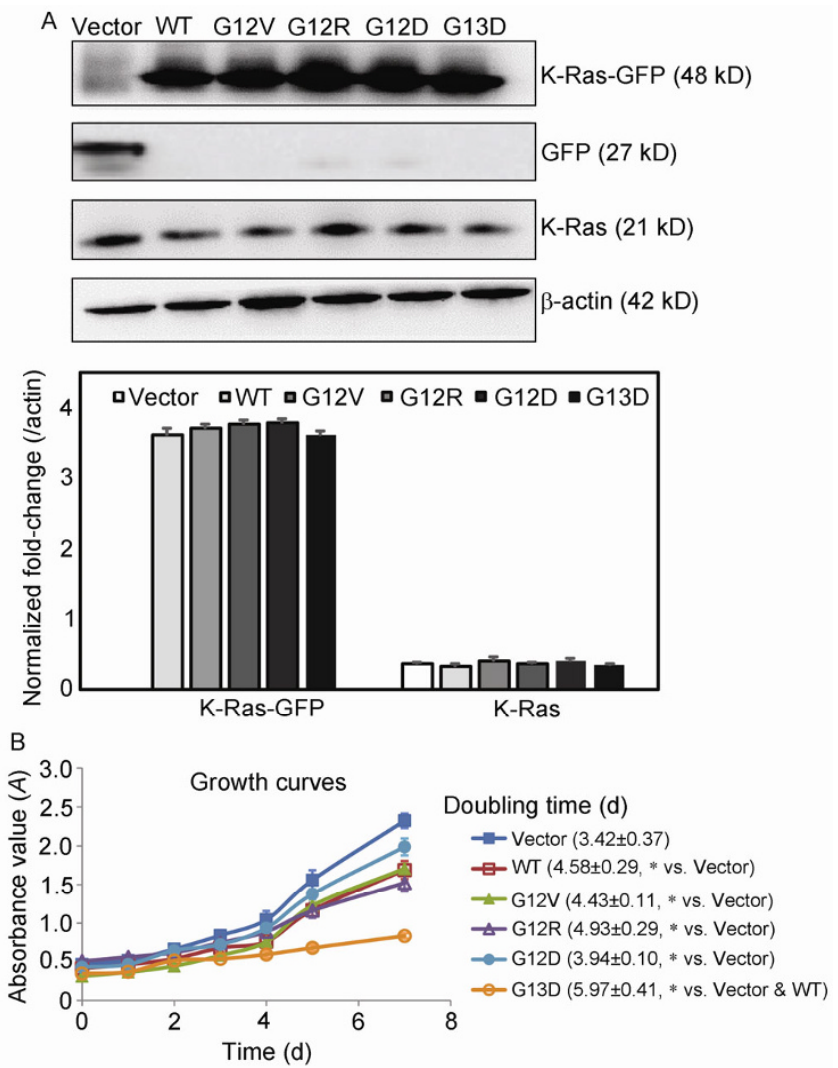

Figure 1 (color online) Expression of K-RAS-pAcGFP1-C3 in Bxpc-3 cells and growth curves of Bxpc-3-derived clones expressing different types of K-ras gene. A, Protein expression of K-Ras-GFP (48 kD), GFP $(27 \mathrm{kD}), \mathrm{K}-\mathrm{Ras}(21 \mathrm{kD})$ and $\beta$-Actin $(42 \mathrm{kD})$ in stable transfected Bxpc-3 cells by Western blot. Densitometric analysis was made by AlphaEaseFC. $\mathrm{B}$, Growth curve of Bxpc-3/vector clone expressing an empty vector ( $\mathbf{a})$, Bxpc-3/Wild type K-Ras ( $\square$ ), Bxpc-3/G12V K-Ras ( $\Delta$ ), Bxpc-3/G12R K-Ras $(\Delta)$, Bxpc-3/G12D K-Ras $(\bullet)$, and Bxpc-3/G13D K-Ras (०).

The vector transfected cells grew a little faster than the K-Ras-pAcGFP1-C3 transfected cells. In all five picked $\mathrm{K}$-ras overexpressed clones, the K-Ras (G13D)-pAcGFP1C3 transfected cells grew slower than all the other clones. In addition, the WT and other mutant clones showed comparable in vitro growth (Figure 1B).

2.2 Drug sensitivities of chemotherapy agents in Bxpc-3 derived clones expressing different K-ras mutations

MTT assay was performed to determine the role of K-ras mutation on the sensitivities of chemotherapy agents. For the EGFR tyrosine kinase inhibitors (EGFR-TKIs), the expression of mutant K-ras was associated with obvious resistance to gefitinib, while showed no reduced sensitivity to erlotinib (Figure 2A and B). For the cytotoxic agents, the mutant clones showed obvious resistance to gemcitabine, 5-FU, and docetaxel compared with the WT clone (Figure $2 \mathrm{C}-\mathrm{E}$ ), whereas the response to cisplatin was not changed (Figure 2F). Moreover, the expression of a specific K-ras 
A Erlotinib

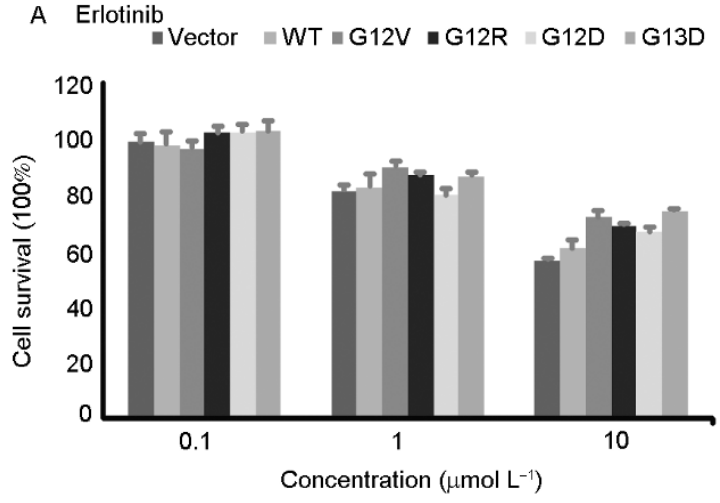

C Gemcitabine -Vector $\quad$ WT $\|\mathrm{G} 12 \mathrm{~V}\| \mathrm{G} 12 \mathrm{R}\|\mathrm{G} 12 \mathrm{D}\| \mathrm{G} 13 \mathrm{D}$

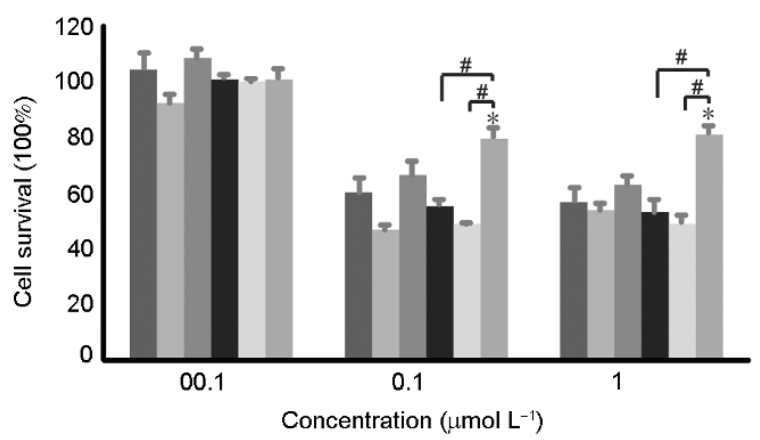

E Cisplatin

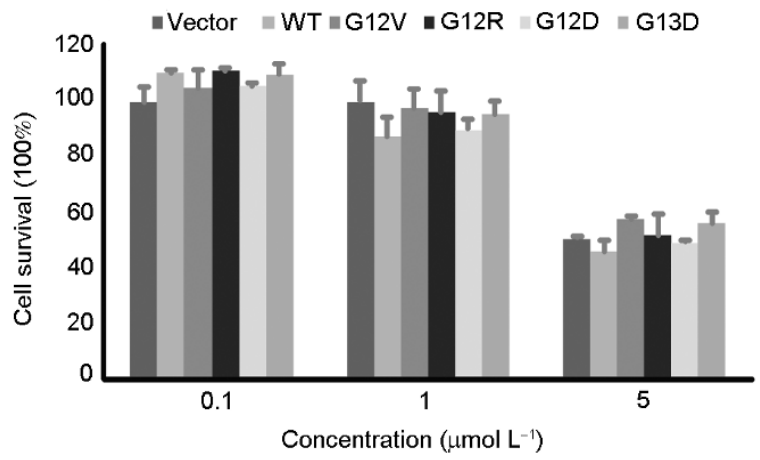

B Gefitinib -Vector $\|\mathrm{WT}\| \mathrm{G} 12 \mathrm{~V}\|\mathrm{G} 12 \mathrm{R}\| \mathrm{G} 12 \mathrm{D} \| \mathrm{G} 13 \mathrm{D}$

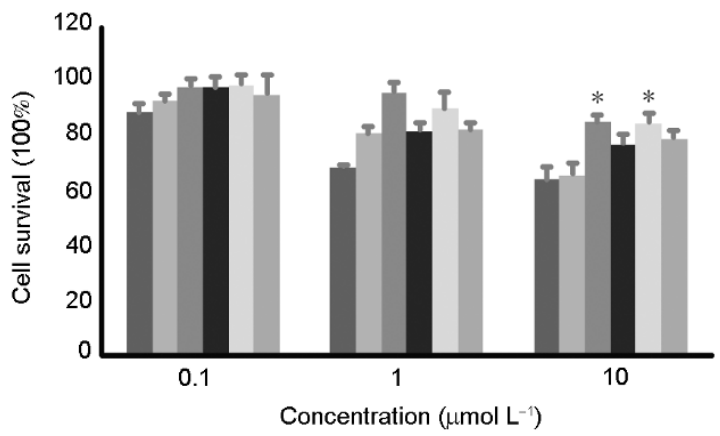

D $\quad 5-\mathrm{FU} \quad \|$ Vector $\|\mathrm{WT}\| \mathrm{G} 12 \mathrm{~V}\|\mathrm{G} 12 \mathrm{R} \approx \mathrm{G} 12 \mathrm{D}\| \mathrm{G} 13 \mathrm{D}$

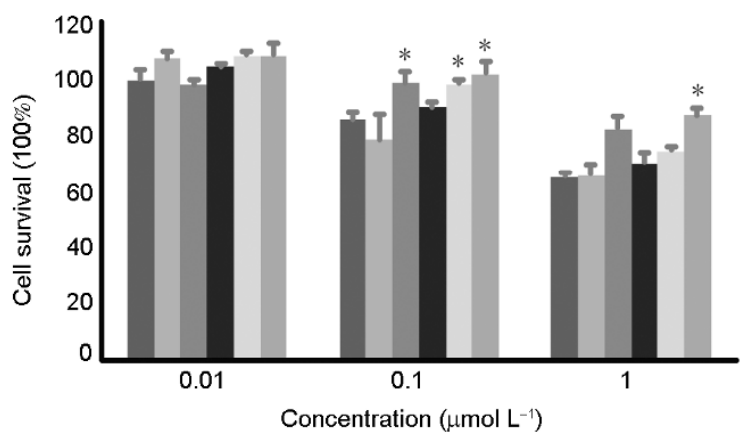

F Docetaxel

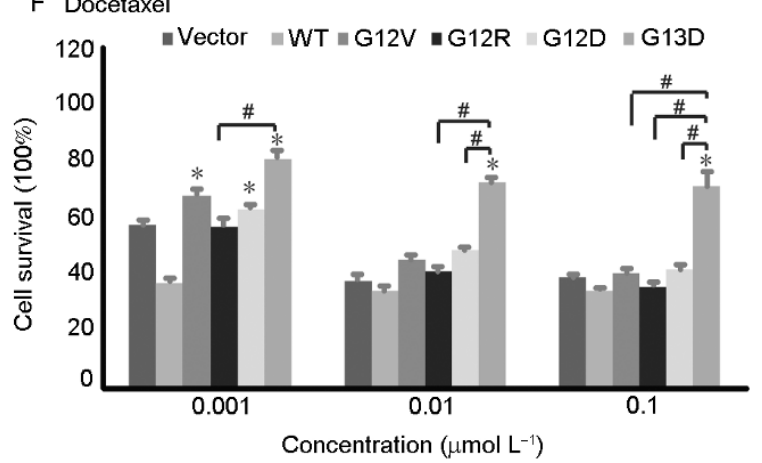

Figure 2 Cell survival of anti-cancer drugs in Bxpc-3-derived clones expressing different types of K-Ras gene. Cells were treated with erlotinib (A), gefitinib (B), gemcitabine (C), 5-FU (D), cisplatin (E), and docetaxel (F) at the indicated drug concentrations. Data are presented as mean \pm SD of three independent experiments. *, $P<0.05$ vs. WT clone and the difference of cell survival $>20 \%$. \#, $P<0.05$ between the two indicated groups and the difference of cell survival $>20 \%$.

mutation induced a different drug sensitivity pattern. Specifically, the expression of G13D was associated with a strong resistance to gemcitabine and docetaxel, whereas the expression of $\mathrm{G} 12 \mathrm{~V}$ resulted in a remarkably reduced response to gefitinib and 5-FU.

2.3 Apoptosis analysis of chemotherapy agents in Bxpc-3 derived clones expressing different K-ras mutations

To determine whether apoptotic cell death is responsible for the reduced sensitivity to gefitinib, 5-FU, and docetaxel, we performed flow cytometry analysis with Annexin V and PI staining. Cells were treated with gefitinib $\left(20 \mu \mathrm{mol} \mathrm{L}^{-1}\right)$, 5-FU $\left(10 \mu \mathrm{mol} \mathrm{L}^{-1}\right)$, and docetaxel $\left(0.01 \mu \mathrm{mol} \mathrm{L}{ }^{-1}\right)$ for $48 \mathrm{~h}$. The results showed that the apoptosis rate of gefitinib in K-ras G12D clone was significantly decreased compared with the WT clone, while the apoptosis rate of 5-FU was obviously lower in K-ras G12V clone (Figure 3). In addition, the induction of apoptosis by docetaxel was reduced in both G12V and G12D mutant cells. These results suggested that the sensitivity to chemotherapy agents was reduced in $\mathrm{K}$-ras mutant cells mainly by decreasing induced tumor 

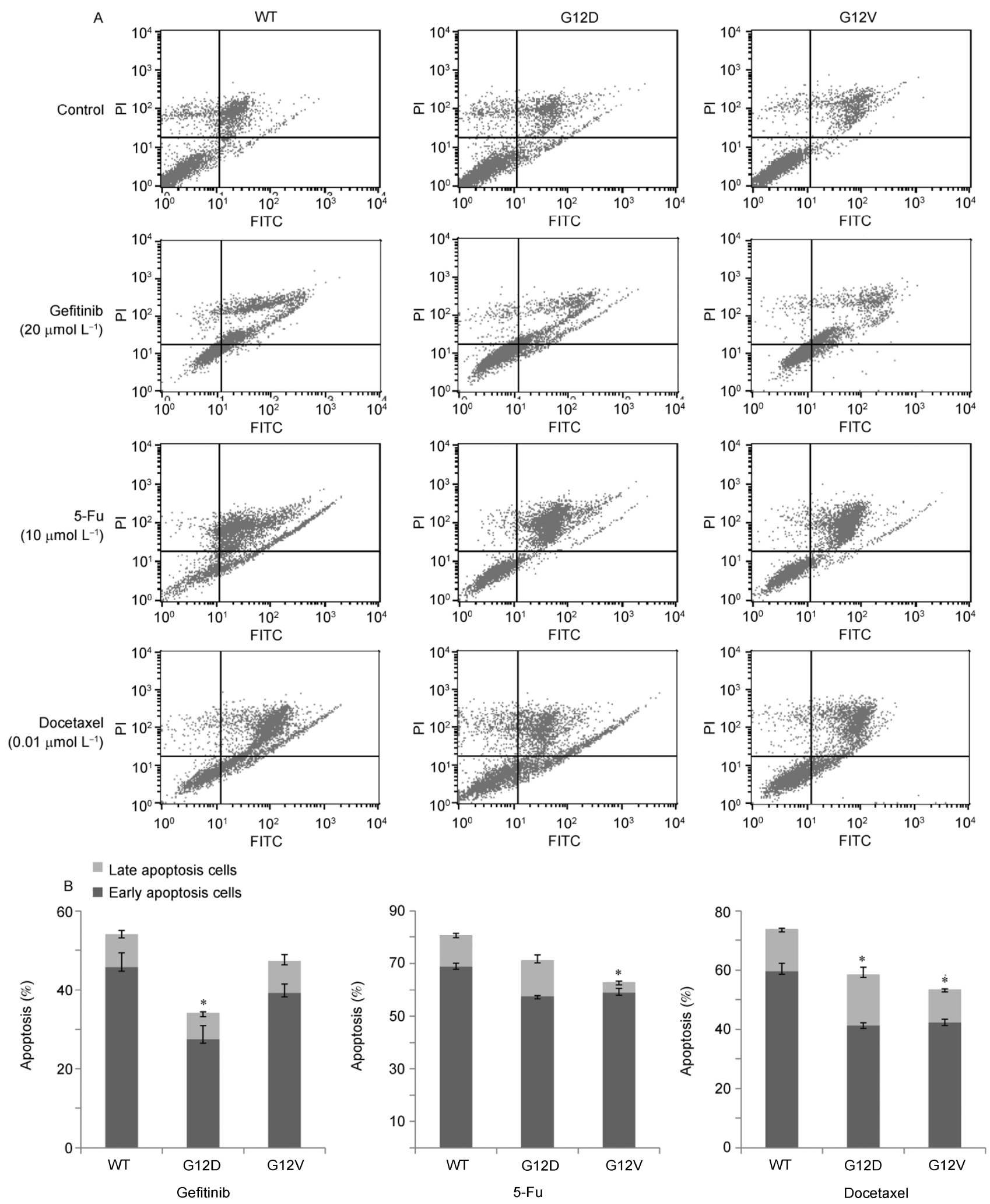

Figure 3 Annexin V and PI staining for apoptosis assay in WT, G12D, and G12V clones after 48 treatment with gefitinib, 5-FU, and docetaxel. A, Apoptosis was assayed by Annexin V and PI staining and fluorescence-activated cell sorting analysis. B, The percentage of early and late apoptotic cells was indicated in the histogram. ${ }^{*}, P<0.05$ vs. WT clone. 
cell apoptosis.

\subsection{K-Ras downstream signaling in Bxpc-3-derived clones expressing different K-ras mutations}

To determine if the overexpression of K-Ras can activate its downstream signaling, we analyzed the levels of p-Akt and p-Erk using Western blot. As shown by the results, the relative expression of p-Akt was up-regulated in K-ras G12V clone, while the levels of p-Erk was significantly higher in K-ras G12V, G12R and G12D clones (Figure 4).

\section{Discussion}

$\mathrm{K}$-ras oncogene mutation is present in $90 \%$ pancreatic adenocarcinoma, but its role in the response of chemotherapy agents is unclear. In this study, we generated K-Ras overexpressing clones with the four most common amino acid substitutions (G12D, G12R, G12V and G13D) from the human pancreatic cancer cell line Bxpc-3 with a WT egfr and K-ras. Except for the K-ras (G13D) clone, the other mutant clones and WT clone showed similar in vitro growth.

As expected, the expression of mutant K-ras induced an excessive activation of downstream signals p-Akt and p-Erk. Specifically, expression of G12V mutant K-ras resulted in activation of p-Akt, while the K-ras G12V, G12R, and G12D clones showed obvious up-regulation of p-Erk than the WT clone. Similar results were reported in many other

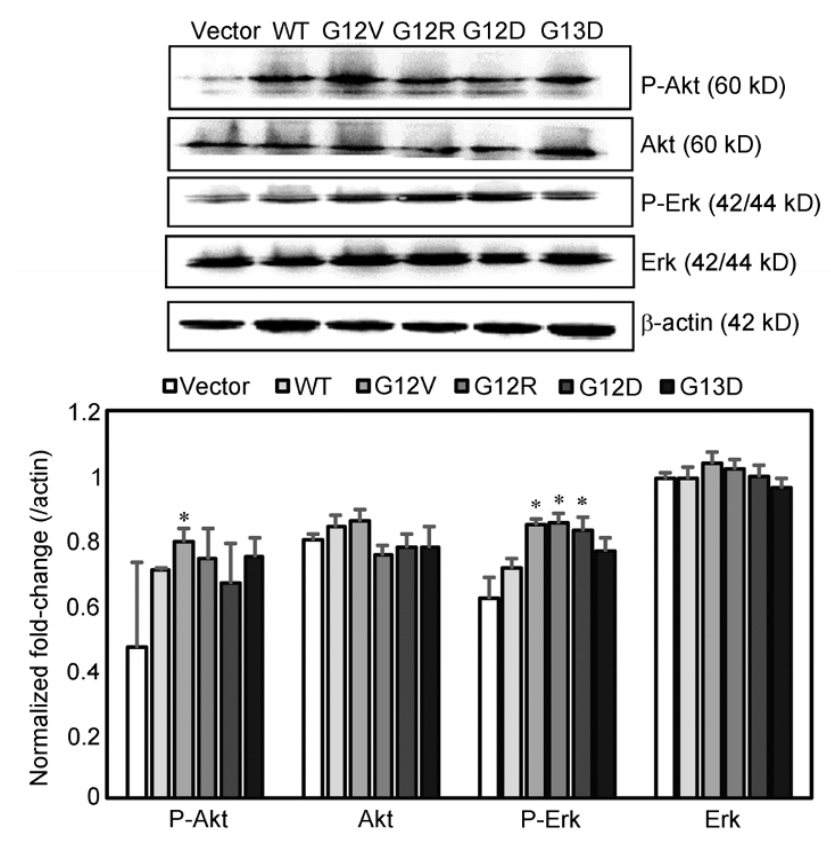

Figure 4 Western blot analysis of K-Ras downstream signaling in Bxpc-3-derived clones expressing different types of K-ras gene. Densitometry quantification was made by AlphaEaseFC. *, $P<0.05$ vs. WT clone. transfected cancer cell lines. Toulany et al. [16] reported that overexpression of G12V mutant K-ras strongly enhanced Akt phosphorylation in human breast cancer SKBr3 cells. Similarly, Minjgee et al. [17] reported that transfection of G12V mutant K-ras not only increased the expression of p-Akt, but also enhanced Erk phosphorylation in head-and-neck cancer FaDu cells. Moreover, expression of G12D mutant K-ras was reported to be able to promote activating of both PI3K and Erk activities in human pancreatic E6/E7/st cells [18]. Further, the present study also revealed the differences in the downstream activation by different types of K-ras mutation in human pancreatic Bxpc-3 cells.

Recent clinical studies have suggested that the K-ras mutation may be a negative predictor of response to the anti-EGFR monoclonal antibody in colorectal cancer, but the influence of K-ras mutations on the therapeutic efficacy of EGFR-TKIs is still debating. In the present study, the sensitivity to gefitinib was reduced in K-ras G12V and G12D mutant Bxpc-3 clones compared with WT clone, while the response to erlotinib was not influenced by K-ras mutation. Gefitinib and erlotinib are both reversibly competitive inhibitors of the tyrosine kinase domain of EGFR, but the present study implied the difference in the anti-cancer effects between the two EGFR-TKIs. The resistance to gefitinib in G12V and G12D clones may be partly explained by the excessive activation of K-Ras downstream signaling p-Akt and/or p-Erk. However, the phosph-Erk was also up-regulated in G12R clone, but the sensitivity to gefitinib was not changed. Therefore, the exact mechanism for the difference in the sensitivity to gefitinib in different types of K-ras mutant cells may warrant further study.

In our study, we also compared the sensitivity to the cytotoxic agents in WT or different mutant clones. Compared with the WT clone, drug resistance to gemcitabine was observed in the G13D clone, while the reduced sensitivity to 5-FU and docetaxel was found in the G12V, G12D, and G13D clones. However, there was no influence of K-ras mutation on the response to cisplatin. This phenomenon may be partly due to the increased phosph-Akt and/or phosph-Erk in mutant clones. As Baneriee et al. [19] reported, the reduction in p-Erk contributed to the reduced sensitivity of pancreatic ductal adenocarcinoma (PDAC) to gemcitabine. Over-expressed p-Erk was also reported to enhance the drug resistance to 5-FU [20] and docetaxel [21]. More interestingly, the expression of a specific K-ras mutation induced a different sensitivity pattern to 5-FU, docetaxel and gemcitabine in Bxpc-3 cells. Specifically, the G13D mutant clone showed a strong resistance to gemcitabine, 5-FU and docetaxel. Additionally, the expression of G12V and G12D mutant K-ras was associated with a remarkably reduced response to 5-FU and docetaxel. The Annexin V/PI staining assay also demonstrated that the apoptosis of 5-FU, and docetaxel was also reduced in K-ras G12D and G12V clones. Previous study also demonstrated 
that different types of K-ras mutation could also affect drug sensitivity in non-small-cell lung cancer cells [22]. But the response pattern to cisplatin and gemcitabine in non-smallcell lung cancer was different from the pattern in pancreatic cancer. The relationship between K-ras mutation and chemotherapy response may be different depending on tumor types, which needs further investigation.

In conclusion, we successfully constructed the recombinant eukaryotic expression plasmids containing different types of mutant K-ras genes. The mutant K-ras genes were stably expressed in Bxpc-3 cells. Expression of mutant K-ras significantly reduced the sensitivity of Bxpc-3 cells to gefitinib, 5-FU, docetaxel and gemcitabine, whereas showed no effect on erlotinib and cisplatin. More interestingly, different types of K-ras mutations could lead to a different pattern of drug sensitivity. Our study has provided preliminary data for further investigation on the effect of different K-ras mutations on the clinical efficacy of chemotherapeutic agents in pancreatic cancer. This may be important for the selection of effective clinical treatment.

This work was supported by National Natural Science Foundation of China (81102459), the Fundamental Research Funds for the Central Universities, Key Laboratory for the Chemistry and Molecular Engineering of Medicinal Resources (Guangxi Normal University), Ministry of Education of China (CMEMR2012-B07), and Doctoral Fund of Ministry of Education of China (20130071110069).

1 Almoguera C, Shibata D, Forrester K, Martin J, Arnheim N, Perucho M. Most human carcinomas of the exocrine pancreas contain mutant c-K-ras genes. Cell, 1988, 53: 549-554

2 Hanlon L, Avila JL, Demarest RM, Troutman S, Allen M, Ratti F, Rustgi AK, Stanger BZ, Radtke F, Adsay V, Long F, Capobianco AJ, Kissil JL. Notch1 functions as a tumor suppressor in a model of K-ras-induced pancreatic ductal adenocarcinoma. Cancer Res, 2010, 70: 4280-4286

3 Johnson L, Mercer K, Greenbaum D, Bronson RT, Crowley D, Tuveson DA, Jacks T. Somatic activation of the K-ras oncogene causes early onset lung cancer in mice. Nature, 2001, 410: 1111-1116

4 Heidorn SJ, Milagre C, Whittaker S, Nourry A, Niculescu-Duvas I, Dhomen N, Hussain J, Reis-Filho JS, Springer CJ, Pritchard C, Marais R. Kinase-dead BRAF and oncogenic RAS cooperate to drive tumor progression through CRAF. Cell, 2010, 140: 209-221

5 Martin TD, Samuel JC, Routh ED, Der CJ, Yeh JJ. Activation and involvement of Ral GTPases in colorectal cancer. Cancer Res, 2011, 71: 206-215

6 Shen L, Nishioka T, Guo J, Chen C. Geminin functions downstream of p53 in K-ras-induced gene amplification of dihydrofolate reductase. Cancer Res, 2012, 72: 6153-6162

7 Yoon J, Koo KH, Choi KY. MEK1/2 inhibitors AS703026 and AZD6244 may be potential therapies for K-RAS mutated colorectal cancer that is resistant to EGFR monoclonal antibody therapy. Cancer Res, 2011, 71: 445-453
8 Zhao L, Vogt PK. Helical domain and kinase domain mutations in p110alpha of phosphatidylinositol 3-kinase induce gain of function by different mechanisms. Proc Natl Acad Sci USA, 2008, 105: 2652-2657

9 Neuzillet C, Hammel P, Tijeras-Raballand A, Couvelard A, Raymond E. Targeting the Ras-ERK pathway in pancreatic adenocarcinoma. Cancer Metastasis Rev, 2013, 32: 147-162

10 Baynes RD, Gansert J. KRAS mutational status as a predictor of epidermal growth factor receptor inhibitor efficacy in colorectal cancer. Am J Ther, 2009, 16: 554-561

11 Tsao MS, Aviel-Ronen S, Ding K, Lau D, Liu N, Sakurada A, Whitehead M, Zhu CQ, Livingston R, Johnson DH, Rigas J, Seymour L, Winton T, Shepherd FA. Prognostic and predictive importance of p53 and RAS for adjuvant chemotherapy in non small-cell lung cancer. J Clin Oncol, 2007, 25: 5240-5247

12 Capelletti M, Wang XF, Gu L, Graziano SL, Kratzke RA, Strauss GM, Maddaus M, Green MR, Vokes EE, Janne PA. Impact of KRAS mutations on adjuvant carboplatin/paclitaxel in surgically resected stage IB NSCLC: CALGB 9633. J Clin Oncol, 2010, 28: 7008

13 Pickard M, Sherrington P, Brett M, Green B, Howelevans A, Smith D, Poston G, Kinsella A. Mutation in the K-ras gene at codon 12 does not correlate with disease progression in colorectal carcinoma patients treated with 5-FU/folinic acid biomodulated chemotherapy. Int J Oncol, 1996, 9: 1301-1306

14 Rosty C, Chazal M, Etienne MC, Letoublon C, Bourgeon A, Delpero JR, Pezet D, Beaune P, Laurent-Puig P, Milano G. Determination of microsatellite instability, p53 and K-RAS mutations in hepatic metastases from patients with colorectal cancer: relationship with response to 5-fluorouracil and survival. Int J Cancer, 2001, 95: $162-167$

15 Xiao J, Chen LW, Chen JX, Lin HY, Huang B. Construction of a recombinant eukaryotic expression plasmid containing human PDLIM2 gene and its biological activity. Plasmid, 2011, 66: 106-111

16 Toulany M, Schickfluss TA, Eicheler W, Kehlbach R, Schittek B, Rodemann HP. Impact of oncogenic K-RAS on YB-1 phosphorylation induced by ionizing radiation. Breast Cancer Res, 2011, 13: R28

17 Minjgee M, Toulany M, Kehlbach R, Giehl K, Rodemann HP. K-RAS(V12) induces autocrine production of EGFR ligands and mediates radioresistance through EGFR-dependent Akt signaling and activation of DNA-PKcs. Int J Radiat Oncol Biol Phys, 2011, 81: 1506-1514

18 Campbell PM, Groehler AL, Lee KM, Ouellette MM, Khazak V, Der CJ. K-Ras promotes growth transformation and invasion of immortalized human pancreatic cells by Raf and phosphatidylinositol 3-kinase signaling. Cancer Res, 2007, 67: 2098-2106

19 Banerjee J, Al-Wadei HA, Schuller HM. Chronic nicotine inhibits the therapeutic effects of gemcitabine on pancreatic cancer in vitro and in mouse xenografts. Eur J Cancer, 2013, 49: 1152-1158

20 Liu S, Wang J, Niu W, Liu E, Wang J, Peng C, Lin P, Wang B, Khan AQ, Gao H, Liang B, Shahbaz M, Niu J. The beta6-integrinERK/MAP kinase pathway contributes to chemo resistance in colon cancer. Cancer Lett, 2013, 328: 325-334

21 Jeong EK, Lee SY, Jeon HM, Ju MK, Kim CH, Kang HS. Role of extracellular signal-regulated kinase (ERK) $1 / 2$ in multicellular resistance to docetaxel in MCF-7 cells. Int J Oncol, 2010, 37: 655-661

22 Garassino MC, Marabese M, Rusconi P, Rulli E, Martelli O, Farina G, Scanni A, Broggini M. Different types of K-Ras mutations could affect drug sensitivity and tumour behaviour in non-small-cell lung cancer. Ann Oncol, 2011, 22: 235-237

Open Access This article is distributed under the terms of the Creative Commons Attribution License which permits any use, distribution, and reproduction in any medium, provided the original author(s) and source are credited. 